\title{
Estudo descritivo do inventário do treino técnico-desportivo do tenista: resultados parciais segundo o 'ranking'
}

\author{
Marcos A. A. Balbinotti ${ }^{1}$ \\ Carlos A. A. Balbinotti ${ }^{2}$ \\ António T. Marques ${ }^{3}$ \\ Adroaldo C. A. Gaya ${ }^{2}$
}

https://doi.org/10.5628/rpcd.05.01.49

\author{
${ }^{1}$ Universidade do Vale do Rio dos Sinos \\ São Leopoldo, RS, Brasil \\ ${ }^{2}$ Universidade Federal do Rio Grande do Sul, Escola \\ Superior de Educação Física, Porto Alegre, RS, Brasil \\ ${ }^{3}$ Universidade do Porto, Faculdade de Ciências do Desporto \\ e de Educação Física, Portugal
}

\begin{abstract}
A descriptive study of the "Technical Training Questionnaire for Tennis Players": partial results according to ranking.

The technical development of young tennis players is obtained with the exercise of sets of techniques, which are required for the optimal performance: techniques of definition (GD) and preparation (GP). The goal of this study was investigate how the technical training of young tennis players from Brazil (13 to 16 years of age), who participated in competitions of the Brazilian Tennis Federation, was performed during the year of 2003. Further, we wanted to describe possible differences between players ranked among the 10 best players in Brazil (TR), and players not ranked among the 10 best players in Brazil (TS). After applying the Tennis Technical Questionnaire (5), a significant difference was found in the frequency of training between GP e GD [TR $\left(t_{(63)}=\right.$ 13.6; $p=0.0001)$; TS $(t=10.6$; $g l=165) p=0.0001)]$. There was a significant difference $(p<0.01)$ between the two groups of athletes; the GP are trained more frequently $(p<0.01)$. There was a significant difference when TR and TS are compared, as GP are trained more frequently than GD. These results suggest a precocious specialization of the technical training performed by young tennis players.
\end{abstract}

A formação técnico-desportiva do jovem tenista é constituída principalmente pelo exercício de um grupo de jogadas exigidas para o desempenho na quadra de jogo: Golpes de Definição (GD) e Golpes de Preparação (GP). Este estudo teve como objetivo investigar como foi realizado o treino técnico-desportivo de jovens tenistas brasileiros (13 - 16 anos), que participaram do circuito nacional de competições da Confederação Brasileira de Tênis (CBT) no ano de 2003. Ainda, objetivamos descrever possíveis diferenças entre o treino dos tenistas posicionados no ranking entre os 10 melhores do país (TR) e tenistas não posicionados entre os 10 melhores do país (TS). Após aplicação do Inventário do Treino Técnico-desportivo do Tenista (ITTT), observou-se diferença significativa na freqüência de treinamento dos grupos de golpes GP e GD [TR $\left(t_{(63)}=\right.$ $13,6 ; p=0,0001)$; TS $(t=10,6 ; \mathrm{gl}=165) \mathrm{p}=0,0001)]$. Existe diferença significativa $(\mathrm{p}<0,01)$ entre os grupos de golpe; os GP são treinados com mais freqüência $(\mathrm{p}<0,01)$. Foi constatada diferença significativa em favor dos GP quando TR e TS são comparados. Estes resultados sugerem que o treino dos tenistas é realizado de forma precoce.

Palavras-chave: estudo descritivo, treino técnico-desportivo, tenistas, ranking.
Key Words: descriptive study, technical training, tennis players, ranking. 


\section{INTRODUÇÃO}

O Brasil tem se destacado no cenário desportivo internacional pelos excelentes resultados obtidos por seus atletas em competições de alto nível. O tenista Gustavo Kuerten é um exemplo de atleta que desde o ano de 1997 coleciona títulos conquistados nos principais torneios do Circuito Profissional de Tênis. A escola de formação de tenistas brasileiros, no entanto, há mais de 10 anos não revela outro atleta deste nível. Será que o treino técnico-desportivo dos jovens tenistas brasileiros está sendo orientado de forma inquestionável?

O estudo tem por objetivo investigar como foi realizado o treino técnico-desportivo de jovens tenistas brasileiros (13 - 16 anos) e, principalmente, apresentar as diferenças encontradas no treino dos tenistas posicionados no ranking entre os 10 melhores do país (TR) em relação aos demais tenistas sem ranking (TS), que participaram do circuito nacional de competições da Confederação Brasileira de Tênis (CBT) no ano de 2003. A faixa etária dos tenistas selecionados representa uma fase crítica de transição na carreira desportiva dos jovens, na qual estabelece as possibilidades de uma carreira profissional de atleta. Salienta-se que os maiores destaques no ranking brasileiro da categoria estão presentes na população estudada (8 tenistas entre os 10 melhores em suas respectivas categorias).

O treino técnico-desportivo do tenista resume-se, fundamentalmente, no exercício de um grupo de jogadas que permitam o aprendizado e o desenvolvimento de todas as ações exigidas para o bom desempenho do tenista em quadra $(41,21)$. Para tanto, foram selecionadas 12 jogadas elementares, divididas em 6 Golpes de Preparação (GP) e 6 Golpes de Definição (GD). Os GP constituem-se numa série de golpes preparatórios para dar início às jogadas ofensivas, enquanto os GD são aqueles golpes utilizados pelo tenista para definir os pontos em disputa $(21,40,41)$.

A construção dos pressupostos para o rendimento desportivo deverá fazer-se fundamentalmente no quadro de uma formação motora e desportiva não especializada, no qual assente-se nos princípios da diversidade da atividade e da formação multilateral (26). Percebe-se a importância do controle do treinamento juvenil baseado num plano caracterizado pela amplitude das ações, em oposição às orientações para o desempenho e treinamento de alto nível (11, 22, 28, 42).

De acordo com Ramos (37), é muito importante para a formação técnico-desportiva do jovem a aquisição dos padrões elementares de execução em sua plenitude, pois muitas vezes verifica-se que os praticantes chegam ao escalão sênior com deficiências ao nível de fundamentos técnicos. Isso ocorre, fundamentalmente, quando há um desequilíbrio na proposta de treino em favor de GP ou até mesmo de GD. Fato que caracteriza o treino técnico-desportivo especializado precocemente (TTEP), pois os tenistas adultos de alto nível costumam especializar-se através do exercício de um grupo específico de jogadas para a obtenção de melhores resultados nas competições (21). O TTEP é, na realidade, um dos sérios problemas que emergem do treino desportivo infanto-juvenil. Especializar precocemente um atleta significa potencializar sua preparação desportiva no sentido de orientá-la de uma forma unilateral prematuramente e, ainda, forçando os ritmos e incrementos das cargas de treino (27).

Com o objetivo de investigar a possível ocorrência do TTEP no treino técnico-desportivo de jovens tenistas brasileiros, Balbinotti e Balbinotti (4) desenvolveram o "Inventário do Treino Técnico-desportivo do Tenista” (ITTT). Aplica-se este instrumento para identificar a freqüência de treino dos GD e GP durante os exercícios realizados na quadra de tênis. Este estudo tem a finalidade de aprofundar os dados descritivos gerais, conforme o ranking do tenista, divulgado pela Confederação Brasileira de Tênis (CBT), relativo ao ano de 2003. Vários autores (2, 9, $13,20,21,25,41)$ recomendam a importância do treino equilibrado de todas as jogadas que compõem GD e GP para a formação integral da técnica desportiva dos jovens tenistas. Considerando que para o modelo adulto de competição de alto nível recomenda-se o treino de um grupo específico de jogadas $(13,20,21)$, a identificação do TTEP ocorrerá se os resultados indicarem que há uma freqüência de treino desproporcional de um grupo de jogadas em detrimento de outras.

Espera-se que os resultados deste estudo possam contribuir para uma melhor estruturação do treino técnico-desportivo destes jovens tenistas. 


\section{MATERIAL E MÉTODOS \\ Procedimento}

O recrutamento dos sujeitos foi realizado durante as competições oficiais da Confederação Brasileira de Tênis no ano de 2003. O ITTT foi aplicado após a detalhada apresentação dos objetivos deste estudo e a formal concordância assinada (consentimento informado) por parte dos jovens tenistas (ou responsáveis) que, nos intervalos das competições, o responderam. Menos de $5 \%$ dos convidados se negaram a responder. Não houve qualquer forma de pressão para que eles o respondessem e, já que seus nomes não eram pedidos, eles estavam assegurados da confidencialidade de suas respostas. O tempo necessário para responder ao ITTT foi de aproximadamente 10 minutos.

\section{Sujeitos}

Considerando as colocações de Maguire e Rodgers (24) com respeito às problemáticas associadas à seleção dos elementos constitutivos de uma amostra aleatória em pesquisas de psicologia e/ou educação, uma amostra não probabilística - 32 meninos (50\%) e 32 meninas (50\%) com idades entre 13 e 16 anos $(\bar{X}=14,52 ; \mathrm{DP}=1,03)$; todos os tenistas brasileiros participantes do circuito nacional Infanto-juvenil foi convenientemente utilizada para responder aos objetivos desta pesquisa. Este grupo de 64 jovens atletas foi dividido em dois: os TR (tenistas posicionados entre as 10 primeiras colocações no ranking nacional de sua categoria); e os TS (demais tenistas participantes do circuito nacional de competições). Destaca-se que a amostra contou com a participação de 8 dos 10 melhores tenistas de sua respectiva categoria: até 14 anos e até 16 anos (masculino e feminino). Portanto, numa população máxima de 40 atletas TR, considerando 4 categorias, 32 atletas participaram da pesquisa.

\section{Instrumento}

"Inventário do Treino Técnico-desportivo do Tenista" (ITTT). Elaborado por Balbinotti e Balbinotti (5), o ITTT avalia a freqüência no treino de 12 iniciativas de jogadas consideradas fundamentais para o desenvolvimento equilibrado e harmonioso do treino técnico-desportivo de tenistas. Estas iniciativas de jogadas foram divididas em duas dimensões distintas, conforme a teoria preconizava.
A primeira, Golpes de Definição (GD), consta de 6 itens que avaliam a freqüência do treino de golpes indefensáveis. A segunda, Golpes de Preparação (GP), consta de 6 itens que avaliam a freqüência do treino de golpes preparatórios para a iniciativa do ataque em busca da definição do ponto. Os jovens envolvidos na pesquisa responderam ao ITTT conforme uma escala bipolar, de tipo Likert, em 5 pontos, sendo 1 "pouquíssima freqüência" e 5 "muitíssima freqüência” de treino.

Quanto às qualidades psicométricas do ITTT, foram realizadas análises de itens (estudo da fidedignidade) e análises fatoriais (estudo da validade de construto). Estes estudos podem ser pormenorizadamente observados em Balbinotti e Balbinotti $(6,7,8)$. Entretanto, com o objetivo de demonstrar os graus de fidedignidade e validade, serão apresentados neste momento apenas os resultados mais significativos, a fim de garantir ao leitor que este instrumento apresenta qualidades psicométricas satisfatórias. Quanto às questões de fidedignidade, realizaram-se as análises correlacionais (Correlação mediana 0,43 para a dimensão GD e de 0,42 para a dimensão GP), onde se pode garantir a homogeneidade das dimensões. Os resultados alpha de Cronbach encontrados (0,81 para ambas as dimensões) sustentam a hipótese da consistência interna do ITTT (18). Nenhum item, se retirado, aumentaria o alpha. A partir destes resultados, pode-se concluir que o ITTT é um instrumento preciso em sua medida.

Quanto às questões de validade de construto, foram realizadas, num primeiro momento, três análises que justificam a aplicação do modelo fatorial para esse conjunto de dados: matriz do determinante das correlações $(0,00008)$, K-M-O $(0,72)$ e o teste de esfericidade de Bartlett (821,76; $\mathrm{p}<0,0001)$. Seus respectivos resultados garantem a adequação no uso do modelo fatorial para esse conjunto de dados. Sendo assim, uma análise fatorial de componentes principais, com rotação varimax, foi conduzida e seus resultados demonstram uma adequada interpretação em dois fatores (que explicam 53,2\% da variância). Estes fatores estão perfeitamente em concordância com as dimensões teoricamente apresentadas. A força encontrada das saturações fatoriais (Sat $>0,52$ ) e das comunalidades (Com $>0,28$ ) sustentam as conclusões de se estar medindo o construto teórico que se desejou medir. 


\section{RESULTADOS}

As análises descritivas realizadas estão conforme a sugestão de alguns dos principais e/ou atuais autores da área da psicometria e dos métodos quantitati$\operatorname{vos}(1,3,12,14,15,16,23,31,33,34,35,36,38$, 44). Assim, realizou-se uma série de estudos descritivos e seus resultados estão apresentados abaixo.

\section{Estudo dos casos extremos}

Conforme os gráficos de bigodes (36), observa-se que na dimensão GP (ver Figura 1) não há indicações de casos extremos. A presença ocorre, apenas, na dimensão GD, para o grupo TS (ver Figura 2). Na realidade, trata-se de um caso que estaria puxando a média para baixo. Mesmo que os valores das médias com e sem casos extremos (ver Tabela 1) sejam nominalmente diferentes (e favorável à média com casos extremos), aplicando um teste de comparação de médias $\left(F_{(1,62)}=3,93 ; p=0,519\right)$ para variâncias homogêneas $\left(F_{\text {Levene }}=3,47 ; \mathrm{p}=0,451\right)$, verificouse que não há diferenças significativas $(\mathrm{p}>0,05)$ entre elas. Tais resultados são estimações importantes e auxiliam a decisão de não desconsiderar os casos extremos no restante das análises. Sendo assim, mesmo que a Tabela 1 apresente os resultados pormenorizados segundo a existência (ou não) de casos extremos, de agora em diante serão analisados apenas os casos "com", inclusive porque na realidade, eles existem.

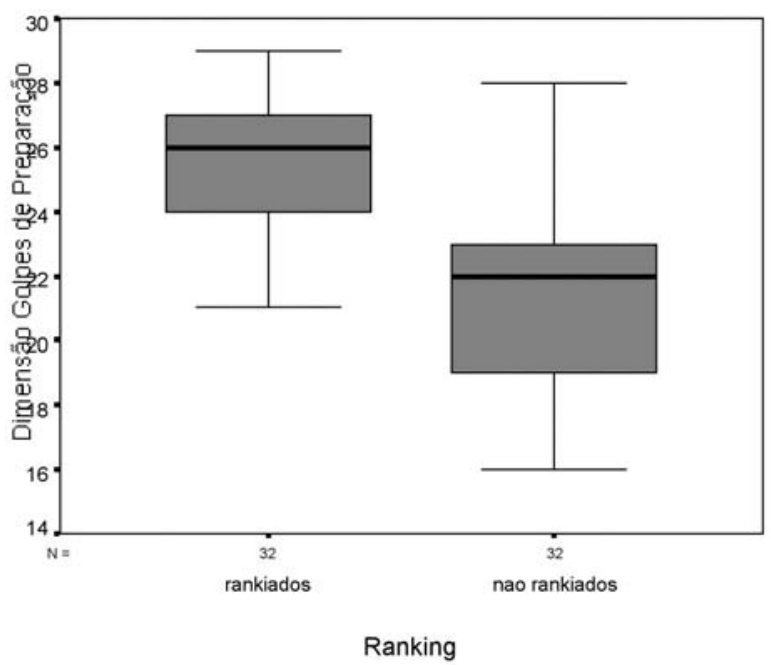

Figura 1: Demonstração gráfica dos casos extremos, por grupo de ranking, na dimensão $G P$.

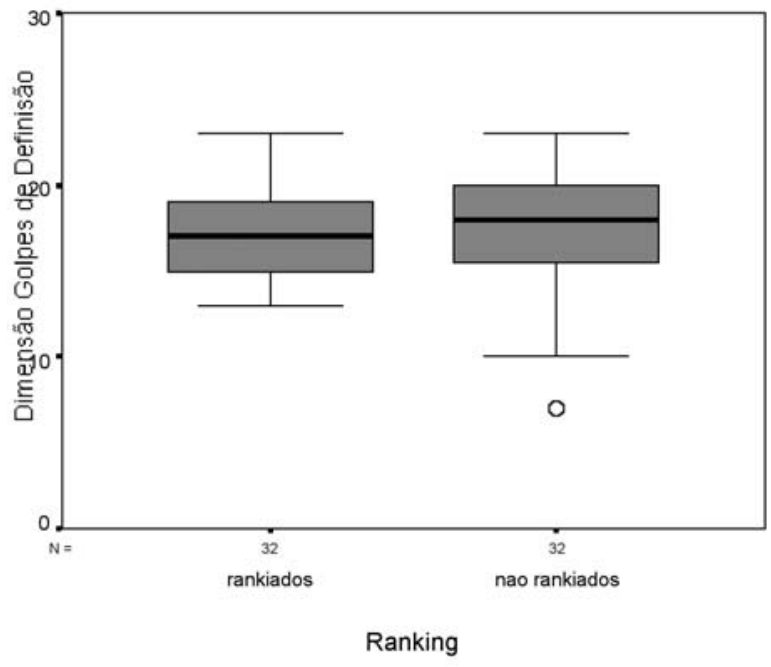

Figura 2: Demonstração gráfica dos casos extremos, por grupo de ranking, na dimensão $G D$. 
Tabela 1: Análises descritivas segundo a existência ou não de casos extremos, por grupo de ranking, para cada dimensão em estudo.

\begin{tabular}{|c|c|c|c|c|c|c|c|c|c|c|c|c|c|c|c|c|}
\hline \multirow{3}{*}{ Dimensão } & \multirow{3}{*}{ Ranking } & \multirow{3}{*}{$\begin{array}{l}\text { C. } \\
\text { E. }^{1}\end{array}$} & \multirow{3}{*}{$\mathrm{n}$} & \multicolumn{7}{|c|}{ Tendência Central e Dispersão } & \multicolumn{6}{|c|}{ Distribuição } \\
\hline & & & & \multirow{2}{*}{$\begin{array}{l}\bar{X} \\
(\sigma)\end{array}$} & \multirow{2}{*}{$\bar{X}_{5 x}$} & \multirow{2}{*}{$M_{\text {ediana }}$} & \multirow{2}{*}{$M_{\text {oda }}$} & \multicolumn{2}{|c|}{ Amplitude } & \multirow{2}{*}{$\sigma_{(X)^{2}}^{2}$} & \multicolumn{2}{|c|}{ Normalidade } & \multicolumn{2}{|c|}{ Assimetria } & \multicolumn{2}{|c|}{ Achatamento } \\
\hline & & & & & & & & $M_{\text {inimal }}$ & $M_{\text {aximal }}$ & & S.W & Sig. & Skewness & $\mathrm{EP}_{5}^{3}$ & Kurtosis & $\mathrm{EP}_{\mathrm{k}}^{4}$ \\
\hline \multirow{4}{*}{ GP } & \multirow[t]{2}{*}{ R } & Com & 32 & $\begin{array}{c}25,5 \\
{[2,0]}\end{array}$ & 25,4 & 26 & 26 & 21 & 29 & 0,36 & 0,958 & 0,352 & $-0,278$ & 0,41 & $-0,339$ & 0,81 \\
\hline & & Sem & -. & .. & .. & .. &.$\cdot$ & .* & .. & .. & .. & .. & .. & .. & .. & .. \\
\hline & $N \cdot R$ & Com & 32 & $\begin{array}{c}21,4 \\
(2,8)\end{array}$ & 21,3 & 22 & 22 & 16 & 28 & 0,49 & 0,969 & 0,533 & $\cdot 0,017$ & 0,41 & $\cdot 0,078$ & 0,81 \\
\hline & & Sem & .. & .. & .. & .. & .. & .. & .. & .. & .. &.$\cdot$ & .. & .. & .. &.. \\
\hline \multirow{4}{*}{ GD } & \multirow[t]{2}{*}{$\mathrm{R}$} & Com & 32 & $\begin{array}{l}17,3 \\
(2,5)\end{array}$ & 17,2 & 17 & 17 & 13 & 23 & 0,44 & 0,950 & 0,235 & $-0,539$ & 0,41 & $-0,223$ & 0,81 \\
\hline & & Sem &.$\cdot$ & .. & .. & .. &.. & .. & .. & .. & .. & .. & .. & .. & .. & .. \\
\hline & \multirow{2}{*}{$N \cdot R$} & Com & 32 & 17,2 & 17,4 & 17 & 18 & $?$ & 23 & 0,68 & 0,933 & 0,084 & 0,842 & 0,41 & 0,480 & 0,81 \\
\hline & & Sem & 31 & 17,6 & $17, ?$ & 18 & 18 & 10 & 23 & 0,62 & 0,940 & 0,207 & $-0,607$ & 0,42 & $-0,031$ & 0,82 \\
\hline
\end{tabular}

1 - Casos Extremos; 2 - Erro Padrão da média; 3 - Erro Padrão do Skewness; 4 - Erro Padrão da Kurtosis.

\section{Estudo dos cálculos de tendência central}

Foram realizados diversos cálculos de tendência central (média, média aparada a 5\%, mediana, moda). Todos com dados interessantes a serem descritos e analisados. Das quatro médias obtidas (considerando o fato de que aquela sem os casos extremos foi excluída das análises) pode-se notar que aquelas referentes à dimensão GD são nominalmente menores que as da dimensão GP (ver Tabela 1). Considerando as diferenças nas médias gerais (não controlando a variável ranking) de GP $\left.\overline{\mathrm{X}}_{\mathrm{gp}}=23,45\right)$ e GD $\overline{(\mathrm{X}}_{\mathrm{gd}}=$ $17,25)$, aplicou-se um teste $t$ para amostras pareadas, a fim de verificar os níveis de significância desta diferença $\left(t_{(63)}=26,43 ; p=0,000\right)$. A partir da constatação de uma correlação praticamente nula $(r=0,004$; $\mathrm{p}=0,977)$ entre as duas dimensões estudadas, os resultados indicam uma diferença altamente significativa $(\mathrm{p}<0,001)$ entre as médias da freqüência de treino dos dois grupos de golpes (diferença favorável aos GP).

Para aprofundar a análise inicial, verificou-se a possível existência de diferenças significativas entre as médias na freqüência de treino dos dois grupos de golpes (GP e GD) com a variável "ranking" controla- da. Assim, realizou-se um ANOVA One-Way para a análise do $\mathrm{GP}\left(\mathrm{F}_{(1,62)}=43,7 ; \mathrm{p}=0,000\right)$ e do GD $(\mathrm{F}$ $=0,013 ; p=0,908)$, diagnosticando-se também os níveis de homogeneidade das variâncias ( $p>0,05$; para ambas as dimensões). Os respectivos resultados indicam não haver diferenças significativas ( $p>$ $0,05)$ entre as médias, obtidas para cada grupo de ranking, na freqüência de treino da dimensão GD. Entretanto, quando se analisa especificamente a dimensão GP, percebe-se uma diferença altamente significativa $(p<0,001)$ favorável ao grupo TR (ver Tabela 1). A partir de então, é possível referir que (mesmo que as médias propriamente ditas sejam nominalmente próximas), em média, os jovens TR treinam mais intensamente GP do que os TS. As implicações decorrentes destes resultados serão interpretadas oportunamente.

Mantendo o ranking constante e verificando as diferenças entre os grupos de golpes (GP e GD), nota-se que, tanto no caso dos TR $\left(t_{(63)}=13,6 ; p=0,000\right)$ quanto dos TS $(t=10,6 ; \mathrm{gl}=165 ; \mathrm{p}=0,000)$, há diferenças altamente significativas $(p<0,01)$ quanto ao treino dos golpes. Essa diferença é favorável aos GP, independente do ranking. Isso significa que, 
em média, a maior parte do tempo de treino da técnica desportiva do tenista é dedicada aos GP. E isso vale tanto para os TR quanto para os TS.

Quanto às análises das médias aparadas a $5 \%$, das medianas e das modas, pode-se dizer que são absolutamente comparáveis às médias aritméticas (ver Tabela 1). Esta constatação irá reforçar o estudo dos cálculos de distribuição. Sendo assim, torna-se necessário o estudo pormenorizado das estatísticas de distribuição (assimetria e achatamento) para justificar os índices de normalidade. Porém, por uma questão de seqüência regular, antes de se apresentar o estudo das estatísticas de distribuição, será apresentado o estudo das estatísticas de dispersão.

\section{Estudo dos cálculos de dispersão}

Os cálculos de dispersão realizados (desvio-padrão, amplitude, erro-padrão da média) servem para nos dar uma precisa informação sobre os níveis de variabilidade dos dados trabalhados nesse estudo.

Embora a variância não tenha sido apresentada na Tabela 1, pode-se conhecê-la apenas multiplicando o desvio-padrão por ele mesmo (36). Inicialmente, sublinha-se que os desvios-padrão variaram de 2,0 a 3,9 , independente da dimensão estudada (desconsiderando os dados sem casos extremos). Pode-se constatar, por um lado, que os desvios não abusivos (considerando as grandezas das médias aritméticas), explicam a homogeneidade das variâncias medidas anteriormente. Por outro lado, a análise dos desviospadrão com e sem casos extremos (na dimensão GD, para o sexo feminino) deixa claro que tais casos não chegavam a aumentar a variabilidade dos dados.

Com certeza, a pequena diferença nominal existente não chegou a ser percebida de forma relevante. No caso da amplitude, pode-se observar que em nenhuma dimensão se encontrou freqüência máxima, o que significa que ninguém respondeu 5 nas 6 questões de cada dimensão. $\mathrm{E}$ os pequenos valores nominais encontrados no caso do erro-padrão da média indicam existir uma ínfima variabilidade dos dados coletados em cada uma das duas dimensões estudadas e em cada um dos dois grupos de ranking controlados.

\section{Estudo dos cálculos de distribuição}

Os três cálculos de distribuição efetuados (normalidade, assimetria e achatamento) servem para dar uma noção precisa da diagramação dos dados (freqüências) conforme a teoria da curva normal. $\mathrm{Na}$ realidade, deve-se atentar ao fato de que os níveis de significância apresentados pelo teste S-W (todos com $p>0,05)$ demonstram que as distribuições são absolutamente normais. Trata-se de um resultado animador no sentido de que, teoricamente, se esperavam distribuições normais. Principalmente, se considerarmos as colocações de Pestana e Gageiro (36), quando afirmam que em amostras não grandes esse dado torna-se imprescindível. Sendo assim, conforme os dados apresentados na Tabela 1 e representados nas Figuras 3, 4, 5 e 6, não há problemas de distribuição amostral. Isso significa não ser necessária nenhuma espécie de transformação dos dados brutos, com o objetivo de normalizar os resultados.

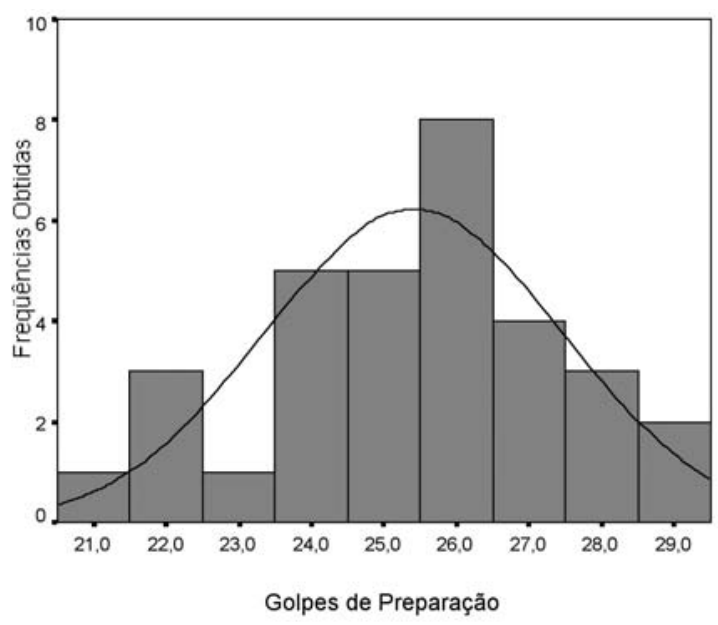

Figura 3: Histograma com curva normal: GP, rankiados.

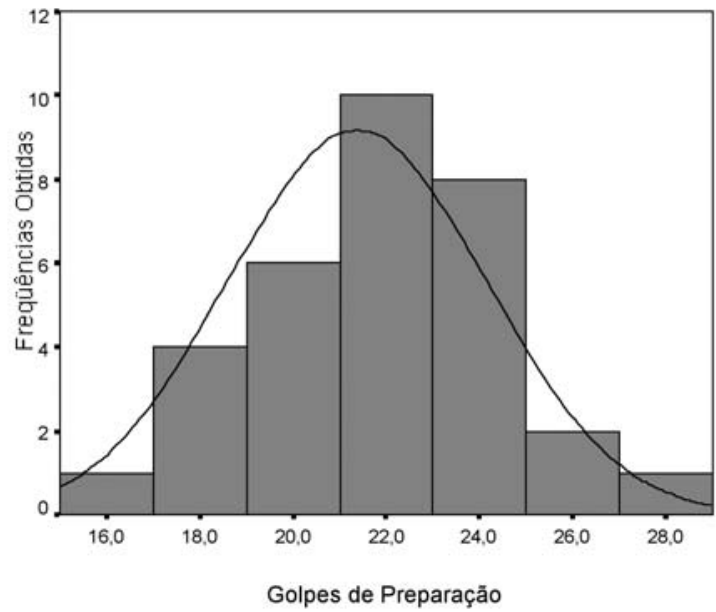

Figura 4: Histograma com curva normal: GP, não-rankiados. 


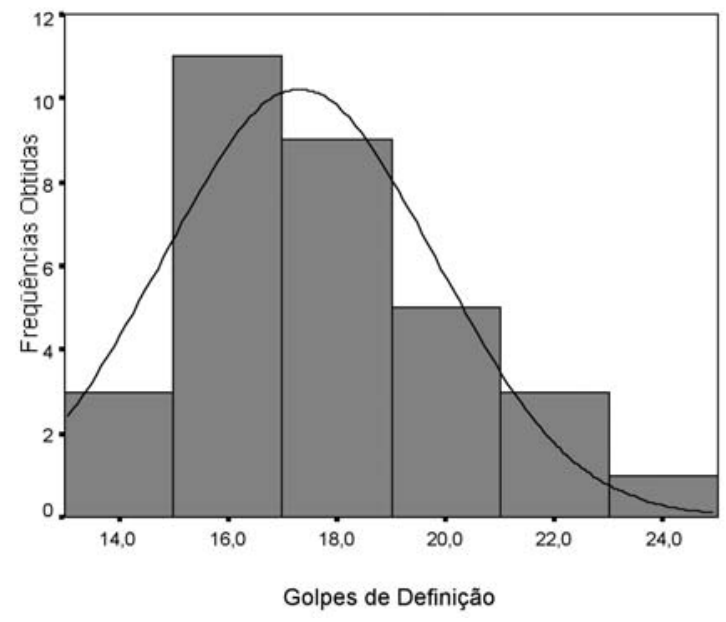

Figura 5: Histograma com curva normal: GD, rankiados.

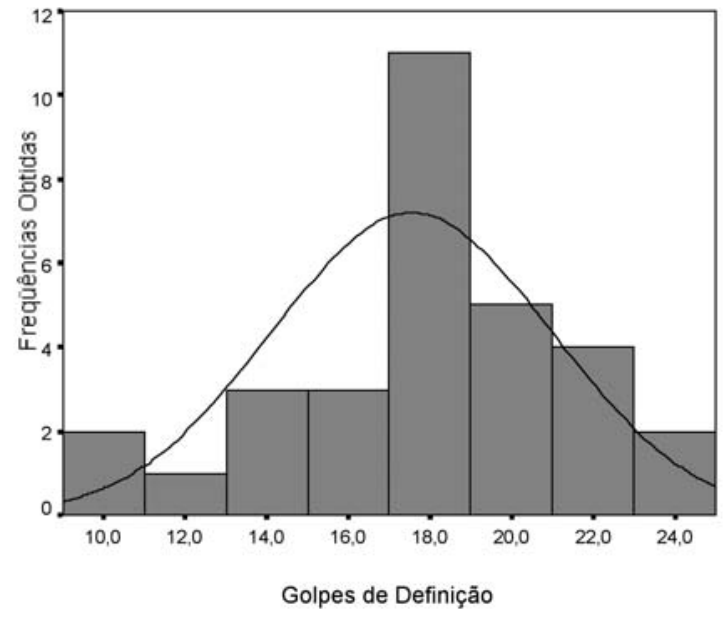

Figura 6: Histograma com curva normal: GD, não-rankiados.

\section{Estudo dos cálculos de percentis, decis e quartis}

Diversos autores $(1,3,12)$ apontam para a importância dos cálculos de tendência não-central realizados (percentis, decis e quartis). Trata-se de uma importante ferramenta de análise do pesquisador. É essa análise que ajuda o pesquisador a decidir a localização (ou a posição) de um sujeito dentro de seu grupo (31) de pares. A Tabela 2 descreve, de forma pormenorizada, diversas possíveis localizações de sujeitos, conforme seus escores no ITTT e, inclusive, seu grupo de ranking.

Tabela 2: Análises dos limites percentílicos.

\begin{tabular}{|c|c|c|c|c|c|c|c|c|c|c|c|c|c|c|c|}
\hline \multirow{4}{*}{ Dimensão } & \multirow{4}{*}{ Ranking } & \multirow{4}{*}{$\mathrm{N}$} & \multicolumn{13}{|c|}{ Análises Descritivas } \\
\hline & & & \multicolumn{13}{|c|}{ Tendência não Central } \\
\hline & & & \multicolumn{13}{|c|}{ Percentis, Quartis e Decis } \\
\hline & & & 1 & 5 & 10 & 20 & 25 & 40 & 50 & 60 & 75 & 80 & 90 & 95 & 99 \\
\hline & $\mathrm{R}$ & 32 & 21 & 21,65 & 22 & 24 & 24 & 25 & 26 & 26 & 27 & 27 & 28 & 29 & 25 \\
\hline \multicolumn{16}{|l|}{ GP } \\
\hline & $N \cdot R$ & 32 & 16 & 16,65 & 17 & 19 & 19 & 21 & 22 & 22,5 & 23 & 23,4 & 24,7 & 26,7 & 28 \\
\hline \multirow{3}{*}{ GD } & $\mathrm{R}$ & 32 & 13 & 13,65 & 14,3 & 15 & 15 & 16 & 17 & 18 & 19 & 19 & 21 & 22,35 & 23 \\
\hline & & & & & & & & & & & & & & & \\
\hline & $N-R$ & 32 & 7 & 8,95 & 10,6 & 13,6 & 15,25 & 17,2 & 18 & 18 & 20 & 21 & 22 & 23 & 23 \\
\hline
\end{tabular}

Para interpretar corretamente os valores obtidos na Tabela 2, cabe salientar o exemplo de um atleta TR que obtém um escore de 21 pontos na dimensão GP, que posiciona-se exatamente no primeiro quartil (Percentil $1_{1}$ ). Isso significa que ele treina apenas cerca de $1 \%$ mais freqüente que seus pares e, conseqüente- mente, $99 \%$ menos freqüente que outros atletas TR. Transpondo-se essa mesma pontuação para um atleta TS (o que causaria um aumento importante para Percentil $_{40}$ ), ainda na dimensão GD, significaria que $60 \%$ de seus pares treinariam com mais freqüência do que ele. Mantendo ainda o mesmo escore (21 
pontos), no caso de atletas TR, para a dimensão GD, este resultado estaria no limite de uma classificação percentílica muito superior (Percentil ${ }_{90}$ ). Tal resultado significaria que $90 \%$ de seus pares treinariam menos freqüente do que esse jovem. Mas, sem dúvida, se fosse um jovem atleta TS que obtivesse um escore de 21 na dimensão GD, estaria classificado como superior (Percentil $1_{80}$ ), o que não seria tão bom quanto seu parceiro TR da mesma dimensão (GD). Tais percentis podem constituir-se em possíveis preditores de resultados positivos ou negativos, embora pesquisas específicas necessitem ser conduzidas para confirmar (ou não) essa hipótese. Cabe salientar que, se verdadeira for essa hipótese, seria possível conhecer um dos preditores de resultados positivos, sem que se fizesse necessário entrar no processo de treino especializado precocemente.

\section{DISCUSSÃO DOS RESULTADOS}

O principal objetivo desta investigação foi apresentar como foi realizado o treino técnico-desportivo de jovens tenistas brasileiros (13 - 16 anos) posicionados entre os 10 melhores do ranking oficial da CBT em relação aos demais tenistas que participam das competições oficiais no ano de 2003.

$\mathrm{O}$ primeiro resultado relevante encontrado neste estudo foi com relação a aderência maior ao treino dos GP em comparação aos GD, para ambos os grupos. Trata-se, portanto, de um primeiro indício real de treino técnico-desportivo especializado precocemente (TTEP). O fato de ocorrer uma maior dedicação ao treino dos GP deve ser em virtude da busca de regularidade no controle da bola na quadra, já que tanto os tenistas TR como os TS procuram adquirir consistência nas jogadas para a preparação do ataque. Outra razão possível decorre do piso com características lentas, onde são disputadas as competições. Os GP são mais eficientes nestas condições, pois se torna mais difícil definir os pontos através da potência e da precisão (21).

A busca incessante de resultados positivos nas competições faz com que os tenistas se especializem cada vez mais nas jogadas que trazem vitórias em curto prazo. Priorizar a formação integral da técnica desportiva se constitui num desafio contra uma tendência da orientação para a obtenção dos resultados competitivos imediatos $(20,40)$.
A insuficiente dedicação dos tenistas ao treino dos GD constatada neste estudo é preocupante. Este grupo de jogadas exige muita precisão e potência nos golpes, razão pela qual ocorrem os erros com muita freqüência. No entanto, para obter a consistência necessária entre os principais fatores que constituem a performance de alto nível na fase adulta: controle, precisão e potência, Schönborn (41) salienta a necessidade de praticar os exercícios relativos aos GD desde a fase de formação do tenista (13 -16 anos).

Parece evidente que os GD não são estimulados no treino em virtude de sua ineficiência nas competições infanto-juvenis. Os GD se caracterizam fundamentalmente pela precisão e a potência. Tendo em vista que os jovens ainda não possuem a força física nem a velocidade necessárias para impor um golpe com potência suficiente para produzir um golpe indefensável para o adversário (10), os GD acabam sendo pouco indicados para os tenistas que buscam a performance em curto prazo.

De fato, as chances dos tenistas jovens obterem resultados positivos nas competições em curto prazo são maiores se a dedicação for exclusiva ao treino dos GP, pois o jogo se desenvolve numa velocidade mais lenta em comparação com as competições adultas $(41,25)$. No entanto, se houvesse equilíbrio no treino de todas as jogadas (GD e GP), mesmo que o desempenho fosse qualitativamente diferente, ainda assim, teríamos as jogadas mais deficientes em processo de desenvolvimento para que na fase mais adulta pudessem corresponder às expectativas do tenista vencedor.

Estas mesmas razões também devem explicar porque os resultados indicam não haver diferenças significativas $(p>0,05)$ entre as médias obtidas para cada um dos grupos na freqüência de treino da dimensão GD. Este resultado sugere que os tenistas TR alcançam o sucesso nas competições, principalmente em conseqüência de seu talento individual e sua capacidade de absorver melhor o treino a eles submetido; jamais devido a um planejamento sistemático por parte dos responsáveis pelo processo de treino. Entretanto, a diferença altamente significativa encontrada nos resultados referentes aos GP favorável ao grupo dos TR não chega a ser surpreendente, pois este grupo de jogadas é considerado mais efi- 
ciente nas competições infanto-juvenis, onde há maior exigência das qualidades físicas: resistência aeróbica e resistência anaeróbica alática $(17,32,29$, 30 ). Os GP caracterizam-se pelo controle e regularidade no domínio da bola $(20,25)$, tendo em vista que se constituem enquanto golpes de preparação para as jogadas de definição, que por sua vez exigem do tenista potência e precisão. Os tenistas infantojuvenis que treinam os GP com muita freqüência conseguem através da regularidade conduzir os adversários ao erro. Enquanto que nas competições adultas os vencedores são aqueles tenistas com maior eficiência nos GD, em razão da potência física e da maturidade técnica na execução dos golpes, no tênis infanto-juvenil o vencedor será aquele tenista que cometer menos erros. A insuficiente força física dos jovens para imprimir potência nos golpes e a imaturidade da construção técnica dos golpes são as principais limitações para se obter sucesso na performance dos GD.

Percebe-se através dos resultados obtidos neste estudo que a orientação do treino técnico-desportivo segue na direção da busca das vitórias nas competições em curto prazo. O fato dos jovens dedicarem uma maior freqüência de treino aos GP demonstra que não está havendo uma preocupação com aquelas jogadas (GD) que serão determinantes para a obtenção das vitórias nas competições adultas.

A constatação de que a freqüência de treino dos GD não é significativa em comparação aos GP, torna evidente a ocorrência do TTEP. Mesmo considerando que os GD se constituem nas principais jogadas utilizadas no tênis adulto, percebe-se claramente que não há uma preocupação com este fato no treino técnico-desportivo dos jovens atletas brasileiros. Deve haver uma proposta de equilíbrio no treino dos GD e GP. Apesar de vários autores $(19,41,21,40)$ recomendarem a necessidade da formação técnico-desportiva integral nas fases de formação dos jovens tenistas, continua havendo uma tendência significativa na proposta de treino favorável a GP. É possível entender as razões que levam a condução deste processo, pois alguns treinadores consideram as vitórias necessárias para manter a motivação dos jovens no treinamento. Todavia, é injustificável nos tempos atuais que este fato ainda possa ocorrer. As conseqüências negativas em médio e longo prazo são inco- mensuráveis, tendo em vista as limitações na qualidade da performance técnico-desportiva do tenista na fase adulta, período em que os GD se constituem nas principais jogadas do tenista. Talvez, alguns treinadores acreditem que os GD deveriam ser treinados apenas na fase adulta. No entanto, considerando o alto grau de dificuldade na execução dos GD, não é possível acreditar que o trabalho que inicie na fase adulta pode ser bem sucedido. Jamais será possível conseguir o domínio da bola com potência e precisão, principal característica de qualidade da performance de GD, sem a quantidade de repetições exigida para a obtenção da maturidade do gesto técnicodesportivo.

Portanto, os resultados obtidos nesta investigação sugerem o aumento do risco de insucesso destes jovens nas futuras competições de tênis a serem realizadas na fase adulta, considerando que há indicações de que está ocorrendo o TTEP.

\section{CONCLUSÕES}

Conforme os resultados apresentados, conclui-se que a maior dedicação na freqüência de treino de GP em comparação com GD para ambos os grupos sugere a ocorrência do TTEP. Outra conclusão relevante do estudo é o fato de haver uma maior adesão aos GP por parte dos tenistas TR em relação aos TS. Cabe ressaltar que esta conclusão também vai ao encontro de uma proposta de treino técnico-desportivo que busca, essencialmente, o imediatismo das vitórias nas competições em curto prazo, pois não há uma preocupação com o treino de GD por parte dos jogadores TR.

\section{CORRESPONDÊNCIA}

\section{Carlos Adelar Abaide Balbinotti}

Rua Lucas de Oliveira 2507/402

Bairro Petrópolis

Cep. 90460 - 001 Porto Alegre, RS, Brasil

balbijan@terra.com.br 


\section{REFERÊNCIAS BIBLIOGRÁFICAS}

1. Annastasi, A.; Urbina, S. (2000). Testagem Psicológica. Porto Alegre, RS: ArtMed Editora.

2. Applewhaite, C. (1988). Golpes que casi nunca se enseñan pero que siempre se ejecutan. In: J. Arranz; J. Andrade; M. Crespo (Eds.) Publicaciones Real Federación Española de Tenis. III Simpósium Int. Prof. Enseñanza. Marbella, 66-82.

3. Baillargeon, G. (1984). Méthodes Statistiques. Trois-Rivières, PQ: Les Éditions SMG.

4. Balbinotti, C. (2003). A formação técnica do jogador de tênis: um estudo sobre jovens tenistas brasileiros. Tese de doutorado. FCDEF-UP. Porto: Portugal.

5. Balbinotti, C.; Balbinotti, M. (2001). Inventário de Aperfeiçoamento Técnico no Tênis. Porto Alegre, RS: Núcleo de Intervenções em Psicologia do Esporte.

6. Balbinotti, C.; Balbinotti, M. (2002). Estudo da consistência interna do inventário de aperfeiçoamento técnico no Tênis. In: Anais do $9^{\circ}$ Congresso Brasileiro de Psicologia do Esporte. Jundiaí, São Paulo.

7. Balbinotti, C.; Balbinotti, M. (2002). Estudo da validade fatorial do inventário de aperfeiçoamento técnico no Tênis. In: Anais do $9^{\circ}$ Congresso Brasileiro de Psicologia do Esporte. Jundiaí, São Paulo.

8. Balbinotti, C.; Balbinotti, M. (2002). Estudo normativo do inventário de aperfeiçoamento técnico no Tênis: resultados parciais segundo o sexo. In: Anais do $9^{\circ}$ Congresso Brasileiro de Psicologia do Esporte. Jundiaí, São Paulo.

9. Barclay, I. (1995). Singles tactics, doubles tactics. LTA Coaching Department.

10. Blimkie, C.; Sale, D. (1998). Strength development and trainability during childhood. In: E. Van Praagh (Ed.) Pediatric anaerobic performance. Champaign, Illinois: Human Kinetics, 193 - 224.

11. Bompa, T. (2000). Total training for Young Champions. Champaign, Illinois: Human Kinetics.

12. Bryman, A.; Cramer, D. (2000). Quantitative Data Analysis: A guide for social scientists. London: Routledge.

13. Crespo, M.; Milley, D. (1999). Manual para entrenadores avanzados. Canada: International Tennis Federation (ITF).

14. Cronbach, L. (1996). Fundamentos da testagem psicológica. Porto Alegre, RS: Artmed Editora.

15. Dassa, C. (1999). Analyse multidimensionnelle exploratoire et confirmative. Montréal, PQ: Presses de 1'Université de Montréal.

16. Fachel, J.; Camey, S. (2000). Avaliação psicométrica: A qualidade das medidas e o entendimento dos dados. In: J. A. Cunha. Psicodiagnóstico-V. Porto Alegre, RS: Artmed Editora.

17. Falk, B.; Tenembaun, G. (1996). The effectiveness of resistance training in

children. Sports Medicine, 22 (3): $176-186$.

18. Garson, D. (2003). PA 765 Statenotes: An Online Textbook. In: http:// www2.chass.ncsu.edu/garson/pa765/statnote.htm

19. Giménez, A. (1999). Modelos de Enseñanza Deportiva: analisis de dos decadas de investigación. Lecturas: Educación Fisica y Deportes, Revista Digital, 4.

20. Gottfried, B. (1994). How to play no every surface. Tennis 31:46-53.

21. Groppel, J. (1993). Tenis para Jugadores Avançados. Madrid: Gymnos.
22. Harre, D. (1989). El processo Del Entrenamiento Deportivo. Havana, Cuba: Editorial Científico Técnico.

23. Kaplan, R.; Saccuzzo, D. (1997). Psychological Testing: Principals, Applications, and Issues. Boston, MA: Brooks/Coles Publishing Company.

24. Maguire, T.; Rogers, W. (1989). Proposed solutions for nonrandomness in educational research. Canadian Journal of Education 14(2), 170 - 181.

25. Lamarche, P.; Cayer, L. (1993). On Court Training of Modern Tactics. ITF Worldwide Coaches Workshop. Key Biscayne.

26. Marques, A. (1999). Crianças e adolescentes atletas: entre a escola e os centros de treino... entre os centros de treino e a escola! Seminário Internacional Treino de Jovens. Comunicações Apresentadas, 17-30.

27. Marques, A. (1991). A especialização precoce na preparação desportiva. Treino Desportivo, 19:9-15.

28. Matvéiev, L. (1986). Fundamentos do Treino Desportivo. Lisboa: Livros Horizonte.

29. Naugton, G.; Farpour-Lambert, N.; Carlson, J.; Bradney, M.; Van Praagh, E. (2000). Physiological issues surrounding the performance of adolescent athletes. Sports Medicine 30 (5): 309 - 325.

30. Nichols, D.; Sandborn, C.; Bonnick, S. (2001). The effects of resistance training on bone mineral density in adolescent females. Journal of Bone and Mineral and Research 11(1): 466.

31. Pasquali, L. (1999). Instrumentos psicológicos: Manual prático de elaboração. Brasília, DF: LabPAM.

32. Payne, V.; Morrow, J.; Johnson, L. (1997). Resistance training in children and youth. A meta-analysis. Research Quarterly Exercise and Sports 68 (1): 80-88.

33. Pestana, M.; Gageiro, J. (1998). Análise de dados para ciências sociais - A complementaridade do SPSS. Lisboa: Edições Sílabo.

34. Pestana, M.; Gageiro, J. (2000). Análise de dados para ciências sociais. Lisboa: Edições Sílabo.

35. Pestana, M.; Gageiro, J. (2001). Análise de dados para ciências sociais - A complementaridade do SPSS. Lisboa: Edições Sílabo.

36. Pestana, M.; Gageiro, J. (2003). Análise de dados para ciências sociais - A complementaridade do SPSS. Lisboa: Edições Sílabo.

37. Ramos, J. (1999). A formação do jovem praticante. Treino Desportivo 6:14-21.

38. Reis, E. (2000). Estatística descritiva. Lisboa: Edições Sílabo.

39. Rink, J.; French, K.; Tjeerdsma, B. (1996). Foundations for de learning and instructions of sports and games. Journal of Teaching in Physical Education 15: 399-417.

40. Sammel, D. (1995). How to counter specialist style. Coaches Review 7:5-6.

41. Schönborn, R. (1999). Ténis: Entrenamiento Técnico. Madrid: Ediciónes Tudor.

42. Tschiene, P. (1990). En Favor de una Teoria del Entrenamiento Juvenil. Revista Stadium 143: 18-28.

43. Tenenbaum, G.; Levy-Kolker, N.; Sade, S.; Liebermann. D. Lidor, R. (1996). Antecipation and confidence of decisions related to skilled performance. International Journal of Sport Psychology 27 (3): 293-307.

44. Thomas, J.; Nelson, J. (2002). Métodos de pesquisa em atividade física. Porto Alegre, RS: ArtMed Editora. 